\title{
A New System of Multivalued Mixed Variational Inequality Problem
}

\author{
$\mathrm{Xi} \mathrm{Li}^{1}$ and Xue-song $\mathrm{Li}^{2}$ \\ ${ }^{1}$ College of Mathematics and Computer, Xihua University, Chengdu, Sichuan 610039, China \\ ${ }^{2}$ Department of Mathematics, Sichuan University, Chengdu, Sichuan 610064, China \\ Correspondence should be addressed to Xue-song Li; xuesongli78@hotmail.com
}

Received 16 February 2014; Accepted 5 April 2014; Published 16 April 2014

Academic Editor: Fu-quan Xia

Copyright (c) 2014 X. Li and X.-s. Li. This is an open access article distributed under the Creative Commons Attribution License, which permits unrestricted use, distribution, and reproduction in any medium, provided the original work is properly cited.

\begin{abstract}
We consider a new system of multivalued mixed variational inequality problem, which includes some known systems of variational inequalities as special cases. Under suitable conditions, the existence of solutions for the system of multivalued mixed variational inequality problem and the convergence of iterative sequences generated by the generalized $f$-projection algorithm are proved. A perturbational algorithm for solving a special case of multivalued mixed variational inequality problem is formally constructed. The results concerned with the existence of solutions and the convergence of iterative sequences generated by the perturbational algorithm are also given. Some known results are improved and generalized.
\end{abstract}

\section{Introduction}

Variational inequalities are known to be very useful tool to formulate and investigate various network equilibrium problems arising in economic, management, and engineering. An important and useful generalization of the variational inequality is called the mixed variational inequality. This problem was originally considered by Lescarret [1] and Browder [2] in connection with its numerous applications. Konnov and Volotskaya [3] considered rather broad classes of general economic equilibrium problems and oligopolistic equilibrium problems which can be formulated as mixed variational inequality problems.

Recently, some interesting and important problems related to variational inequalities and mixed variational inequalities have been considered by many authors. Chang et al. [4] introduced a generalized system for relaxed cocoercive variational inequalities in Hilbert spaces and established some algorithms for the system. Petrot [5] studied a generalized system for relaxed cocoercive mixed variational inequality problem in Hilbert spaces and found the common solutions for the system using a resolvent operator technique. For more details related to variational inequalities and mixed variational inequalities, we refer to [6-12] and the references therein.

It is well known that projection methods have represented an important tool for solving variational inequalities. In 1994, Alber [13] introduced the generalized projections in uniformly convex and uniformly smooth Banach spaces and studied their properties in detail. Recently, Wu and Huang [14] introduced a new generalized $f$-projection operator in a Banach space, which was a useful tool for solving mixed variational inequality problems. They extended the definition of the generalized projection operators introduced by Alber $[13,15]$ and proved some properties of the generalized $f$ projection operator. Fan et al. [16] presented some basic results for the generalized $f$-projection operator and discussed the existence of solutions and approximation of the solutions for generalized variational inequalities in noncompact subsets of Banach spaces by using iterative schemes. Very recently, Li et al. [17] proved some stability results for the generalized $f$-projection operators with perturbations of constraint sets in Banach spaces.

Inspired and motivated by the previous works mentioned above, in this paper, we introduce a new system of multivalued mixed variational inequality problem in Hilbert spaces. This class of systems includes some known systems of 
variational inequalities as special cases. We construct a new generalized $f$-projection algorithm for solving the system of multivalued mixed variational inequality problem. The existence of solutions and the convergence of iterative sequences generated by the algorithm are presented in this paper. We also construct a perturbational algorithm for solving a special case of multivalued mixed variational inequality problem and give the existence of solutions and the convergence of iterative sequences generated by the perturbational algorithm. Our results improve and generalize some known corresponding ones.

\section{Preliminaries}

Let $H$ be a real Hilbert space with scalar product and norm denoted by $\langle\cdot, \cdot\rangle$ and $\|\cdot\|$, respectively. We recall the concept of the generalized $f$-projector operator, together with its properties. Let $R=(-\infty,+\infty)$, let $K \subset H$ be a closed convex subset, and let $f: K \rightarrow R \cup\{+\infty\}$ be a proper convex and lower semicontinuous functional. Let $G: H \times K \rightarrow R \cup\{+\infty\}$ be a functional defined as follows:

$$
G(x, \xi)=\|x\|^{2}-2\langle x, \xi\rangle+\|\xi\|^{2}+2 \rho f(\xi),
$$

where $\xi \in K, x \in H$, and $\rho$ is a positive number.

Definition 1 (see [14]). Let $H$ be a real Hilbert space, and let $K$ be a nonempty closed and convex subset of $H$. Let $f: K \rightarrow$ $R \cup\{+\infty\}$ be a proper, convex, and lower semicontinuous functional. One says that $P_{K}^{f, \rho}: H \rightarrow 2^{K}$ is a generalized $f$-projection operator if

$$
P_{K}^{f, \rho} x=\left\{u \in K: G(x, u)=\inf _{\xi \in K}(x, \xi)\right\}, \quad \forall x \in H .
$$

Lemma 2 (see $[14,16]$ ). Let $H$ be a real Hilbert space, and let $K$ be a nonempty closed and convex subset of $H$. Let $f$ : $K \rightarrow R \cup\{+\infty\}$ be a proper, convex, and lower semicontinuous functional. Then the following statements hold:

(i) $P_{K}^{f, \rho}$ is a single-valued mapping with nonempty values;

(ii) for all $x \in H, x^{*}=P_{K}^{f, \rho} x$ if and only if

$\left\langle x^{*}-x, y-x^{*}\right\rangle+\rho f(y)-\rho f\left(x^{*}\right) \geq 0, \quad \forall y \in K$

(iii) $P_{K}^{f, \rho}$ is continuous.

Lemma 3 (see [18]). Let $H$ be a real Hilbert space, and let $K$ be a nonempty closed and convex subset of $H$. Let $f$ : $K \rightarrow R \cup\{+\infty\}$ be a proper, convex, and lower semicontinuous functional. Then

$$
\left\|P_{K}^{f, \rho} v-P_{K}^{f, \rho} u\right\| \leq\|v-u\|, \quad \forall v, u \in H .
$$

Let $K_{1}$ and $K_{2}$ be closed convex subsets in $H$. It is known that the Hausdorff distance between $K_{1}$ and $K_{2}$ is defined as follows:

$$
H\left(K_{1}, K_{2}\right)=\max \left\{\sup _{x \in K_{1}} \inf _{y \in K_{2}}\|x-y\|, \sup _{y \in K_{2}} \inf _{x \in K_{1}}\|x-y\|\right\} .
$$

Lemma 4 (see [17]). Let $H$ be a real Hilbert space and let $K$ be a nonempty closed convex subset of $H$. Let $f: H \rightarrow R \cup\{+\infty\}$ be a convex and uniformly continuous mapping. Let $\left\{K_{n}\right\}_{n=0}^{+\infty} \subset$ $H$ be a family of nonempty closed convex subsets such that $f$ is proper on each $K_{n}$ and $H\left(K_{n}, K\right) \rightarrow 0$ as $n \rightarrow+\infty$. Then, for any $x_{0} \in H$,

$$
\lim _{n \rightarrow+\infty} P_{K_{n}}^{f, \rho} x_{0}=P_{K}^{f, \rho} x_{0} .
$$

Lemma 5 (see [19]). Let $\left\{a_{n}\right\}$ and $\left\{b_{n}\right\}$ be two nonnegative real sequences satisfying

$$
a_{n+1} \leq k a_{n}+b_{n}
$$

with $0<k<1$ and $b_{n} \rightarrow 0$. Then $\lim _{n \rightarrow 0} a_{n}=0$.

In order to obtain our results, the following definitions are crucial to us.

Definition 6. Let $T: H \times H \rightarrow H$ be a mapping. $T$ is said to be

(i) $\gamma$-Lipschitz continuous with respect to the first argument, if there exists a constant $\gamma>0$ such that

$$
\|T(x, z)-T(y, z)\| \leq \gamma\|x-y\|, \quad \forall x, y, z \in H ;
$$

(ii) $\mu$-strongly monotone with respect to the first argument, if there exists a constant $\mu>0$ such that

$$
\langle T(x, z)-T(y, z), x-y\rangle \geq \mu\|x-y\|^{2}, \quad \forall x, y, z \in H .
$$

Definition 7 (see [20]). Let $F: H \rightarrow 2^{H}$ be a multivalued mapping. $F$ is said to be $\xi$-H-Lipchitz continuous, if there exists a constant $\xi>0$ such that

$$
H(F(x), F(y)) \leq \xi\|x-y\|, \quad \forall x, y \in H .
$$

\section{The Multivalued Mixed Variational Inequality System}

In this section, we will introduce a new system of multivalued mixed variational inequality in a Hilbert space. Let $H$ be a real Hilbert space, and let $K$ be a nonempty closed convex subset of $H$. Let $S, T: H \times H \rightarrow H$ be two single-valued mappings, let $f: K \rightarrow R \cup\{+\infty\}$ be a proper, convex, and lower semicontinuous mapping, and let $E, F: H \rightarrow 2^{H}$ be two set-valued mappings. We consider the following system of multivalued mixed variational inequality problem: find $(x, y) \in K \times K, u \in E(x)$, and $v \in F(y)$ such that

$$
\begin{array}{ll}
\langle S(x, v), z-x\rangle+f(z)-f(x) \geq 0, & \forall z \in K, \\
\langle T(y, u), z-y\rangle+f(z)-f(y) \geq 0, & \forall z \in K .
\end{array}
$$

If $E=F \equiv I$, where $I$ is the identity mapping, then the problem (11) reduces immediately to the following mixed variational inequality system: find $(x, y) \in K$ such that

$$
\begin{array}{ll}
\langle S(x, y), z-x\rangle+f(z)-f(x) \geq 0, & \forall z \in K, \\
\langle T(y, x), z-y\rangle+f(z)-f(y) \geq 0, & \forall z \in K .
\end{array}
$$


If $K=H, E=F \equiv I, S(x, y)=s A(y, x)+x-y$, and $T(y, x)=r A(x, y)+x-y$ for all $x, y \in H$, where $A: H \times H \rightarrow$ $H$, then the problem (11) is equivalent to finding $(x, y) \in H$ such that

$$
\begin{array}{r}
\langle s A(y, x)+x-y, z-x\rangle+f(z)-f(x) \geq 0, \\
\forall z \in H, \quad s>0, \\
\langle r A(x, y)+y-x, z-y\rangle+f(z)-f(y) \geq 0, \\
\forall z \in H, \quad r>0,
\end{array}
$$

which was studied by Petrot [5].

If $E=F \equiv I, f \equiv 0, S(x, y)=s A(y, x)$, and $T(y, x)=$ $r A(x, y)$ for all $x, y \in H$, where $A: H \times H \rightarrow H$ and $s, r>0$, then the problem (11) is equivalent to finding $(x, y) \in K$ such that

$$
\begin{aligned}
& \langle s A(y, x)+x-y, z-x\rangle \geq 0, \quad \forall z \in K, s>0, \\
& \langle r A(x, y)+y-x, z-y\rangle \geq 0, \quad \forall z \in K, r>0 .
\end{aligned}
$$

The system (14) was studied by Chang et al. [4].

In brief, the system (11) of multivalued mixed variational inequality is more general and includes many systems of variational inequalities as special cases (see $[6-9,21])$.

The property of generalized $f$-projection operator plays an important role in solving the system of multivalued mixed variational inequality problem. From Lemma 2, it is easy to see that the following lemma holds trivially.

Lemma 8. The problem (11) is equivalent to finding $(x, y) \in$ $K \times K, u \in E(x)$, and $v \in F(y)$ such that

$$
\begin{aligned}
& x=P_{K}^{f, \rho_{1}}\left(x-\rho_{1} S(x, v)\right), \\
& y=P_{K}^{f, \rho_{2}}\left(y-\rho_{2} T(y, u)\right),
\end{aligned}
$$

where $\rho_{1}, \rho_{2}>0$.

For any given $\left(x_{0}, y_{0}\right) \in K \times K$, we choose $u_{0} \in E\left(x_{0}\right)$ and $v_{0} \in F\left(y_{0}\right)$. By Nadler Jr. [22], for any $\left(x_{1}, y_{1}\right) \in K \times K$ and $\varepsilon>0$, there exist $u_{1} \in E\left(x_{1}\right)$ and $v_{1} \in F\left(y_{1}\right)$ such that

$$
\begin{gathered}
\left\|u_{0}-u_{1}\right\| \leq(1+\varepsilon) H\left(E\left(x_{0}\right), E\left(x_{1}\right)\right), \\
\left\|v_{0}-v_{1}\right\| \leq(1+\varepsilon) H\left(F\left(y_{0}\right), F\left(y_{1}\right)\right) .
\end{gathered}
$$

Based on (15) and (16), we can construct the following algorithm for solving the problem (11).

Algorithm 9. Assume that $K, S, T, E, F$, and $f$ are the same as in the problem (11). For any given $\left(x_{0}, y_{0}\right) \in K \times K$, $u_{0} \in E\left(x_{0}\right)$, and $v_{0} \in F\left(y_{0}\right)$, we compute $x_{n+1}, y_{n+1}, u_{n+1}$, and $v_{n+1}$ as follows:

$$
\begin{array}{r}
x_{n+1}=\left(1-\alpha_{n}\right) x_{n}+\alpha_{n} P_{K}^{f, \rho_{1}}\left(x_{n}-\rho_{1} S\left(x_{n}, v_{n}\right)\right), \\
y_{n+1}=\left(1-\beta_{n}\right) y_{n}+\beta_{n} P_{K}^{f, \rho_{2}}\left(y_{n}-\rho_{2} T\left(y_{n}, u_{n}\right)\right), \\
u_{n+1} \in E\left(x_{n+1}\right), \\
\left\|u_{n+1}-u_{n}\right\| \leq\left(1+(n+1)^{-1}\right) H\left(E\left(x_{n+1}\right), E\left(x_{n}\right)\right), \\
v_{n+1} \in F\left(y_{n+1}\right), \\
\left\|v_{n+1}-v_{n}\right\| \leq\left(1+(n+1)^{-1}\right) H\left(F\left(y_{n+1}\right), F\left(y_{n}\right)\right), \\
n=0,1,2, \ldots,
\end{array}
$$

where $0<\alpha_{n}, \beta_{n} \leq 1$ are constants.

Let $\left\{K_{n}\right\}_{n=0}^{+\infty}$ be a family of nonempty closed convex subsets in $H$. Now we construct the following perturbational algorithm for the problem (12).

Algorithm 10. Assume that $K, S, T$, and $f$ are the same as in the problem (11). For any given $\left(x_{0}, y_{0}\right) \in K \times K$, we compute $x_{n+1}$ and $y_{n+1}$ as follows:

$$
\begin{array}{r}
x_{n+1}=P_{K_{n}}^{f, \rho_{1}}\left(x_{n}-\rho_{1} S\left(x_{n}, y_{n}\right)\right), \\
y_{n+1}=P_{K_{n}}^{f, \rho_{2}}\left(y_{n}-\rho_{2} T\left(y_{n}, x_{n}\right)\right), \\
n=0,1,2, \ldots .
\end{array}
$$

\section{Existence and Convergence Theorems}

In this section, we will prove the existence of solutions for the problem (11) and the unique existence of solutions for the problem (12), respectively. In addition, we will provide the convergence results of Algorithms 9 and 10, respectively.

Theorem 11. Let $H$ be a real Hilbert space, let $K$ be a nonempty closed convex subset of $H$, and let $f: K \rightarrow R \cup\{+\infty\}$ be a proper, convex, and lower semicontinuous mapping. Let $S: H \times$ $H \rightarrow H$ be $\mu_{1}$-strongly monotone and $r_{1}$-Lipschitz continuous with respect to the first variable and $\tau_{1}$-Lipschitz continuous with respect to the second variable. Let T:H $\mathrm{T} \times \mathrm{H} \rightarrow \mathrm{H}$ be $\mu_{2}$-strongly monotone and $r_{2}$-Lipschitz continuous with respect to the first variable and $\tau_{2}$-Lipschitz continuous with respect to the second variable. Let $E: H \rightarrow C(H)$ be $\xi_{1}-H$-Lipschitz continuous; let $F: H \rightarrow C(H)$ be $\xi_{2}-H$-Lipschitz continuous, where $C(H)$ denotes the collection of all closed subsets of $H$. Suppose that $0<\alpha, \beta \leq 1$, and $\rho_{1}, \rho_{2}>0$ satisfy

$$
\begin{gathered}
\theta_{1}=\sqrt{1-2 \rho_{1} \mu_{1}+\rho_{1}^{2} r_{1}^{2}}<1, \\
\theta_{2}=\sqrt{1-2 \rho_{2} \mu_{2}+\rho_{2}^{2} r_{2}^{2}}<1, \\
\lim _{n \rightarrow+\infty} \alpha_{n}=\alpha, \quad \lim _{n \rightarrow+\infty} \beta_{n}=\beta, \\
\beta \rho_{2} \tau_{2} \xi_{1}<\alpha\left(1-\theta_{1}\right), \quad \alpha \rho_{1} \tau_{1} \xi_{2}<\beta\left(1-\theta_{2}\right) .
\end{gathered}
$$


Then $\left(x_{n}, y_{n}, u_{n}, v_{n}\right)$ generated by Algorithm 9 converges strongly to $(x, y, u, v)$ as $n \rightarrow+\infty$; moreover, $(x, y, u, v)$ is a solution of system (11).

Proof. From Algorithm 9 and Lemma 3, we have

$$
\begin{aligned}
\| x_{n+1}- & x_{n} \| \\
\leq & \left(1-\alpha_{n}\right)\left\|x_{n}-x_{n-1}\right\| \\
& +\alpha_{n} \| P_{K}^{f, \rho_{1}}\left(x_{n}-\rho_{1} S\left(x_{n}, v_{n}\right)\right) \\
& \quad-P_{K}^{f, \rho_{1}}\left(x_{n-1}-\rho_{1} S\left(x_{n-1}, v_{n-1}\right)\right) \| \\
\leq & \left(1-\alpha_{n}\right)\left\|x_{n}-x_{n-1}\right\| \\
& +\alpha_{n}\left\|x_{n}-x_{n-1}-\rho_{1}\left(S\left(x_{n}, v_{n}\right)-S\left(x_{n-1}, v_{n-1}\right)\right)\right\| \\
\leq & \left(1-\alpha_{n}\right)\left\|x_{n}-x_{n-1}\right\| \\
& +\alpha_{n} \rho_{1}\left\|S\left(x_{n-1}, v_{n}\right)-S\left(x_{n-1}, v_{n-1}\right)\right\| \\
& +\alpha_{n}\left\|x_{n}-x_{n-1}-\rho_{1}\left(S\left(x_{n}, v_{n}\right)-S\left(x_{n-1}, v_{n}\right)\right)\right\| .
\end{aligned}
$$

Since $S$ is $\mu_{1}$-strongly monotone and $r_{1}$-Lipschitz continuous with respect to the first variable, we obtain

$$
\begin{aligned}
\| x_{n}- & x_{n-1}-\rho_{1}\left(S\left(x_{n}, v_{n}\right)-S\left(x_{n-1}, v_{n}\right)\right) \|^{2} \\
\leq & \left\|x_{n}-x_{n-1}\right\|^{2} \\
& \quad-2 \rho_{1}\left\langle S\left(x_{n}, v_{n}\right)-S\left(x_{n-1}, v_{n}\right), x_{n}-x_{n-1}\right\rangle \\
& +\rho_{1}^{2}\left\|S\left(x_{n}, v_{n}\right)-S\left(x_{n-1}, v_{n}\right)\right\|^{2} \\
\leq & \left\|x_{n}-x_{n-1}\right\|^{2}-2 \mu_{1} \rho_{1}\left\|x_{n}-x_{n-1}\right\|^{2} \\
& +\rho_{1}^{2} r_{1}^{2}\left\|x_{n}-x_{n-1}\right\|^{2} .
\end{aligned}
$$

The $\xi_{2}-H$-Lipschitz continuity of $F$ yields that

$$
\begin{aligned}
& \left\|S\left(x_{n-1}, v_{n}\right)-S\left(x_{n-1}, v_{n-1}\right)\right\| \\
& \quad \leq \tau_{1}\left\|v_{n}-v_{n-1}\right\| \\
& \quad \leq \tau_{1}\left(1+\frac{1}{n}\right) H\left(F\left(y_{n}\right), F\left(y_{n-1}\right)\right) \\
& \quad \leq \tau_{1}\left(1+\frac{1}{n}\right) \xi_{2}\left\|y_{n}-y_{n-1}\right\| .
\end{aligned}
$$

It follows from inequalities (20)-(22) that

$$
\begin{aligned}
\| x_{n+1}- & x_{n} \| \\
\leq & \left(1-\alpha_{n}\right)\left\|x_{n}-x_{n-1}\right\|+\alpha_{n} \rho_{1} \tau_{1} \xi_{2}\left(1+\frac{1}{n}\right)\left\|y_{n}-y_{n-1}\right\| \\
& +\alpha_{n} \sqrt{1-2 \rho_{1} \mu_{1}+\rho_{1}^{2} r_{1}^{2}}\left\|x_{n}-x_{n-1}\right\| \\
= & \left(1-\alpha_{n}+\alpha_{n} \theta_{1}\right)\left\|x_{n}-x_{n-1}\right\|+a_{n}\left\|y_{n}-y_{n-1}\right\|,
\end{aligned}
$$

where $a_{n}=\alpha_{n} \rho_{1} \tau_{1} \xi_{2}(1+1 / n)$ and $\theta_{1}=\sqrt{1-2 \rho_{1} \mu_{1}+\rho_{1}^{2} r_{1}^{2}}$.
By the assumptions of $T$ and $E$, following very similar arguments from (20)-(23), we have

$$
\begin{aligned}
& \left\|T\left(y_{n-1}, u_{n}\right)-T\left(y_{n-1}, u_{n-1}\right)\right\| \\
& \quad \leq \tau_{2}\left\|u_{n}-u_{n-1}\right\| \\
& \quad \leq \tau_{2}\left(1+\frac{1}{n}\right) H\left(E\left(x_{n}\right), E\left(x_{n-1}\right)\right) \\
& \quad \leq \tau_{2}\left(1+\frac{1}{n}\right) \xi_{1}\left\|x_{n}-x_{n-1}\right\|, \\
& \left\|y_{n}-y_{n-1}-\rho_{2}\left(T\left(y_{n}, u_{n}\right)-T\left(y_{n-1}, u_{n}\right)\right)\right\|^{2} \\
& \leq\left(1-2 \rho_{2} \mu_{2}+\rho_{2}^{2} r_{2}^{2}\right)\left\|y_{n}-y_{n-1}\right\|^{2} .
\end{aligned}
$$

Hence,

$$
\begin{aligned}
\| y_{n+1}- & y_{n} \| \\
\leq & \left(1-\beta_{n}\right)\left\|y_{n}-y_{n-1}\right\|+\beta_{n} \rho_{2} \tau_{2} \xi_{1}\left(1+\frac{1}{n}\right) \\
& \times\left\|x_{n}-x_{n-1}\right\| \\
& +\beta_{n} \sqrt{1-2 \rho_{2} \mu_{2}+\rho_{2}^{2} r_{2}^{2}}\left\|y_{n}-y_{n-1}\right\| \\
= & \left(1-\beta_{n}+\beta_{n} \theta_{2}\right)\left\|y_{n}-y_{n-1}\right\|+b_{n}\left\|x_{n}-x_{n-1}\right\|,
\end{aligned}
$$

where $b_{n}=\beta_{n} \rho_{2} \tau_{2} \xi_{1}(1+1 / n)$ and $\theta_{2}=\sqrt{1-2 \rho_{2} \mu_{2}+\rho_{2}^{2} r_{2}^{2}}$.

Now (23) and (26) imply that

$$
\begin{aligned}
\| x_{n+1}- & x_{n}\|+\| y_{n+1}-y_{n} \| \\
\leq & \left(1-\alpha_{n}+\alpha_{n} \theta_{1}+b_{n}\right)\left\|x_{n}-x_{n-1}\right\| \\
& +\left(1-\beta_{n}+\beta_{n} \theta_{2}+a_{n}\right)\left\|y_{n}-y_{n-1}\right\| \\
\leq & \lambda_{n}\left(\left\|x_{n}-x_{n-1}\right\|+\left\|y_{n}-y_{n-1}\right\|\right),
\end{aligned}
$$

where

$$
\begin{array}{r}
\lambda_{n}=\max \left\{1-\alpha_{n}+\alpha_{n} \theta_{1}+b_{n}, 1-\beta_{n}+\beta_{n} \theta_{2}+a_{n}\right\}, \\
\forall n=1,2, \ldots .
\end{array}
$$

Since $\lim _{n \rightarrow+\infty} \alpha_{n}=\alpha$ and $\lim _{n \rightarrow+\infty} \beta_{n}=\beta$, we know that $\lim _{n \rightarrow+\infty} \lambda_{n}=\lambda$, where

$$
\begin{array}{r}
\lambda=\max \left\{1-\alpha\left(1-\theta_{1}\right)+\beta \rho_{2} \tau_{2} \xi_{1},\right. \\
\left.1-\beta\left(1-\theta_{2}\right)+\alpha \rho_{1} \tau_{1} \xi_{2}\right\} .
\end{array}
$$


It is clear that $0<\lambda<1$ due to (19). Consequently, there exist $N>0$ and $\lambda<\widehat{\lambda}<1$ such that $\lambda_{n} \leq \hat{\lambda}$ for all $n \geq N$. It follows from (27) that

$$
\begin{aligned}
\| x_{m} & -x_{n}\|+\| y_{m}-y_{n} \| \\
& \leq \sum_{k=n}^{m-1}\left(\left\|x_{k+1}-x_{k}\right\|+\left\|y_{k+1}-y_{k}\right\|\right) \\
& \leq \sum_{k=n}^{m-1} \lambda_{k}\left(\left\|x_{k}-x_{k-1}\right\|+\left\|y_{k}-y_{k-1}\right\|\right) \\
& \leq \sum_{k=n}^{m-1} \hat{\lambda}^{k-N}\left(\left\|x_{N+1}-x_{N}\right\|+\left\|y_{N+1}-y_{N}\right\|\right),
\end{aligned}
$$

for all $m>n \geq N$. Letting $n \rightarrow+\infty$ in (30), we obtain $\lim _{n \rightarrow+\infty}\left\|x_{m}-x_{n}\right\|=0$ and $\lim _{n \rightarrow+\infty}\left\|y_{m}-y_{n}\right\|=0$, and so $\left\{x_{n}\right\}$ and $\left\{y_{n}\right\}$ are Cauchy sequences in $K$. Therefore, there exist $x, y \in K$ such that $\lim _{n \rightarrow+\infty} x_{n}=x$ and $\lim _{n \rightarrow+\infty} y_{n}=$ $y$.

From inequalities (22) and (24), we know that $\left\{u_{n}\right\}$ and $\left\{v_{n}\right\}$ are both Cauchy sequences in $H$, and so there exist $u, v \in$ $H$ such that $\lim _{n \rightarrow+\infty} u_{n}=u$ and $\lim _{n \rightarrow+\infty} v_{n}=v$. Since $u_{n} \epsilon$ $E\left(x_{n}\right)$ and $v_{n} \in F\left(y_{n}\right)$, we have

$$
\begin{aligned}
d(u, E(x)) & =\inf \{\|u-z\|, z \in E(x)\} \\
& \leq\left\|u-u_{n}\right\|+d\left(u_{n}, E(x)\right) \\
& \leq\left\|u-u_{n}\right\|+H\left(E\left(x_{n}\right), E(x)\right) \\
& \leq\left\|u-u_{n}\right\|+\xi_{1}\left\|x_{n}-x\right\| \longrightarrow 0, \\
d(v, F(y)) & =\inf \{\|v-z\|, z \in F(y)\} \\
& \leq\left\|v-v_{n}\right\|+d\left(v_{n}, F(y)\right) \\
& \leq\left\|v-v_{n}\right\|+H\left(F\left(y_{n}\right), F(y)\right) \\
& \leq\left\|v-v_{n}\right\|+\xi_{2}\left\|y_{n}-y\right\| \longrightarrow 0 .
\end{aligned}
$$

Thus, $u \in E(x)$ and $v \in F(y)$. By the continuity of $P_{K}^{f}$, S, and $T$, it follows from (17) that

$$
\begin{aligned}
& x=P_{K}^{f, \rho_{1}}\left(x-\rho_{1} S(x, v)\right), \\
& y=P_{K}^{f, \rho_{2}}\left(y-\rho_{2} T(y, u)\right) .
\end{aligned}
$$

Lemma 8 shows that $(x, y, u, v)$ is a solution of system (11). This completes the proof.

Theorem 12. Let $H$ be a real Hilbert space and let $K$ be a nonempty closed convex subsets of $H$. Let $f: H \rightarrow R \cup$ $\{+\infty\}$ be a convex and uniformly continuous mapping and let $\left\{K_{n}\right\}_{n=0}^{+\infty} \subset H$ be a family of nonempty closed convex subsets such that $f$ is proper on each $K_{n}$ and $H\left(K_{n}, K\right) \rightarrow 0$ as $n \rightarrow+\infty$. Let $S, T$ be the same as in Theorem 11. Suppose that $\rho_{1}, \rho_{2}>0$ are such that

$$
\begin{aligned}
& \sqrt{1-2 \rho_{1} \mu_{1}+\rho_{1}^{2} r_{1}^{2}}+\rho_{2} \tau_{2}<1, \\
& \sqrt{1-2 \rho_{2} \mu_{2}+\rho_{2}^{2} r_{2}^{2}}+\rho_{1} \tau_{1}<1 .
\end{aligned}
$$

Then $\left(x_{n}, y_{n}\right)$ generated by Algorithm 10 converges strongly to $(x, y)$ as $n \rightarrow+\infty$; moreover, $(x, y)$ is the unique solution of system (12).

Proof. Define the norm $\|\cdot\|_{1}$ on product space $H \times H$ by

$$
\|(u, v)\|_{1}=\|u\|+\|v\|, \quad \forall(u, v) \in H \times H .
$$

It is easy to see that $\left(H \times H,\|\cdot\|_{1}\right)$ is a Banach space. Let $G$ : $K \times K \rightarrow K \times K$ be defined by

$$
\begin{array}{r}
G(u, v)=\left(P_{K}^{f, \rho_{1}}\left(u-\rho_{1} S(u, v)\right), P_{K}^{f, \rho_{2}}\left(v-\rho_{2} T(v, u)\right)\right), \\
\forall(u, v) \in H \times H .
\end{array}
$$

For any $\left(u_{1}, v_{1}\right),\left(u_{2}, v_{2}\right) \in H \times H$, it follows from Lemma 3 that

$$
\begin{aligned}
\| G & \left(u_{1}, v_{1}\right)-G\left(u_{2}, v_{2}\right) \| \\
= & \left\|P_{K}^{f, \rho_{1}}\left(u_{1}-\rho_{1} S\left(u_{1}, v_{1}\right)\right)-P_{K}^{f, \rho_{1}}\left(u_{2}-\rho_{1} S\left(u_{2}, v_{2}\right)\right)\right\| \\
& +\left\|P_{K}^{f, \rho_{2}}\left(v_{1}-\rho_{2} T\left(v_{1}, u_{1}\right)\right)-P_{K}^{f, \rho_{2}}\left(v_{2}-\rho_{2} T\left(v_{2}, u_{2}\right)\right)\right\| \\
\leq & \left\|u_{1}-u_{2}-\rho_{1} S\left(u_{1}, v_{1}\right)+\rho_{1} S\left(u_{2}, v_{2}\right)\right\| \\
& +\left\|v_{1}-v_{2}-\rho_{2} T\left(v_{1}, u_{1}\right)+\rho_{2} T\left(v_{2}, u_{2}\right)\right\| \\
\leq & \left\|u_{1}-u_{2}-\rho_{1} S\left(u_{1}, v_{1}\right)+\rho_{1} S\left(u_{2}, v_{1}\right)\right\| \\
& +\rho_{1}\left\|S\left(u_{2}, v_{1}\right)-S\left(u_{2}, v_{2}\right)\right\| \\
& +\left\|v_{1}-v_{2}-\rho_{2} T\left(v_{1}, u_{1}\right)+\rho_{2} T\left(v_{2}, u_{1}\right)\right\| \\
& +\rho_{2}\left\|T\left(v_{2}, u_{1}\right)-T\left(v_{2}, u_{2}\right)\right\| \\
\leq & \left\|u_{1}-u_{2}-\rho_{1} S\left(u_{1}, v_{1}\right)+\rho_{1} S\left(u_{2}, v_{1}\right)\right\|+\rho_{1} \tau_{1}\left\|v_{1}-v_{2}\right\| \\
& +\left\|v_{1}-v_{2}-\rho_{2} T\left(v_{1}, u_{1}\right)+\rho_{2} T\left(v_{2}, u_{1}\right)\right\| \\
& +\rho_{2} \tau_{2}\left\|u_{1}-u_{2}\right\| .
\end{aligned}
$$


By the assumptions imposed on $S$ and $T$, we obtain

$$
\begin{aligned}
& \| u_{1}- u_{2}-\rho_{1} S\left(u_{1}, v_{1}\right)+\rho_{1} S\left(u_{2}, v_{1}\right) \|^{2} \\
& \leq\left\|u_{1}-u_{2}\right\|^{2}-2 \rho_{1}\left\langle S\left(u_{1}, v_{1}\right)-S\left(u_{2}, v_{1}\right), u_{1}-u_{2}\right\rangle \\
&+\rho_{1}^{2}\left\|S\left(u_{1}, v_{1}\right)-S\left(u_{2}, v_{1}\right)\right\|^{2} \\
& \leq\left(1-2 \mu_{1} \rho_{1}+\rho_{1}^{2} r_{1}^{2}\right)\left\|u_{1}-u_{2}\right\|^{2}, \\
&\left\|v_{1}-v_{2}-\rho_{2} T\left(v_{1}, u_{1}\right)+\rho_{2} T\left(v_{2}, u_{1}\right)\right\|^{2} \\
& \leq\left\|v_{1}-v_{2}\right\|^{2}-2 \rho_{2}\left\langle T\left(v_{1}, u_{1}\right)-T\left(v_{2}, u_{1}\right), v_{1}-v_{2}\right\rangle \\
& \quad+\rho_{2}^{2}\left\|T\left(v_{1}, u_{1}\right)-T\left(v_{2}, u_{1}\right)\right\|^{2} \\
& \leq\left(1-2 \mu_{2} \rho_{2}+\rho_{2}^{2} r_{2}^{2}\right)\left\|v_{1}-v_{2}\right\|^{2} .
\end{aligned}
$$

From (36)-(37), we have

$$
\begin{aligned}
& \left\|G\left(u_{1}, v_{1}\right)-G\left(u_{2}, v_{2}\right)\right\| \\
& \leq\left(\sqrt{1-2 \rho_{1} \mu_{1}+\rho_{1}^{2} r_{1}^{2}}+\rho_{2} \tau_{2}\right)\left\|u_{1}-u_{2}\right\| \\
& \quad+\left(\sqrt{1-2 \rho_{2} \mu_{2}+\rho_{2}^{2} r_{2}^{2}}+\rho_{1} \tau_{1}\right)\left\|v_{1}-v_{2}\right\| \\
& \leq k\left\|\left(u_{1}, v_{1}\right)-\left(u_{2}, v_{2}\right)\right\|_{1},
\end{aligned}
$$

where $k=\max \left\{\sqrt{1-2 \rho_{1} \mu_{1}+\rho_{1}^{2} r_{1}^{2}}+\rho_{2} \tau_{2}\right.$, $\left.\sqrt{1-2 \rho_{2} \mu_{2}+\rho_{2}^{2} r_{2}^{2}}+\rho_{1} \tau_{1}\right\}$. It follows assumption (33) that $0<k<1$. This shows that $G: K \times K \rightarrow K \times K$ is a contractive operator, and so there exists a unique $(x, y) \in K \times K$ such that $G(x, y)=(x, y)$. Thus, $(x, y)$ is the unique solution of system (12).

Now we prove that $x_{n} \rightarrow x$ and $y_{n} \rightarrow y$ as $n \rightarrow+\infty$. In fact, it follows from (18) that

$$
\begin{aligned}
\| x_{n+1}- & x \| \\
= & \left\|P_{K_{n}}^{f, \rho_{1}}\left(x_{n}-\rho_{1} S\left(x_{n}, y_{n}\right)\right)-P_{K}^{f, \rho_{1}}\left(x-\rho_{1} S(x, y)\right)\right\| \\
\leq & \left\|P_{K_{n}}^{f, \rho_{1}}\left(x_{n}-\rho_{1} S\left(x_{n}, y_{n}\right)\right)-P_{K_{n}}^{f, \rho_{1}}\left(x-\rho_{1} S(x, y)\right)\right\| \\
& +\left\|P_{K_{n}}^{f, \rho_{1}}\left(x-\rho_{1} S(x, y)\right)-P_{K}^{f, \rho_{1}}\left(x-\rho_{1} S(x, y)\right)\right\|, \\
\| y_{n+1}- & y \| \\
= & \left\|P_{K_{n}}^{f, \rho_{2}}\left(y_{n}-\rho_{2} T\left(y_{n}, x_{n}\right)\right)-P_{K}^{f, \rho_{2}}\left(y-\rho_{2} T(y, x)\right)\right\| \\
= & \left\|P_{K_{n}}^{f, \rho_{2}}\left(y_{n}-\rho_{2} T\left(y_{n}, x_{n}\right)\right)-P_{K_{n}}^{f, \rho_{2}}\left(y-\rho_{2} T(y, x)\right)\right\| \\
& +\left\|P_{K_{n}}^{f, \rho_{2}}\left(y-\rho_{2} T(y, x)\right)-P_{K}^{f, \rho_{2}}\left(y-\rho_{2} T(y, x)\right)\right\| .
\end{aligned}
$$

Following very similar arguments from (36)-(38), we have

$$
\begin{aligned}
&\left\|P_{K_{n}}^{f, \rho_{1}}\left(x_{n}-\rho_{1} S\left(x_{n}, y_{n}\right)\right)-P_{K_{n}}^{f, \rho_{1}}\left(x-\rho_{1} S(x, y)\right)\right\| \\
& \quad+\left\|P_{K_{n}}^{f, \rho_{2}}\left(y_{n}-\rho_{2} T\left(y_{n}, x_{n}\right)\right)-P_{K_{n}}^{f, \rho_{2}}\left(y-\rho_{2} T(y, x)\right)\right\| \\
& \leq k\left(\left\|x_{n}-x\right\|+\left\|y_{n}-y\right\|\right) .
\end{aligned}
$$

It follows from (39)-(40) that

$$
\left\|x_{n+1}-x\right\|+\left\|y_{n+1}-y\right\| \leq k\left(\left\|x_{n}-x\right\|+\left\|y_{n}-y\right\|\right)+b_{n},
$$

where

$$
\begin{aligned}
b_{n}= & \left\|P_{K_{n}}^{f, \rho_{1}}\left(x-\rho_{1} S(x, y)\right)-P_{K}^{f, \rho_{1}}\left(x-\rho_{1} S(x, y)\right)\right\| \\
& +\left\|P_{K_{n}}^{f, \rho_{2}}\left(y-\rho_{2} T(y, x)\right)-P_{K}^{f, \rho_{2}}\left(y-\rho_{2} T(y, x)\right)\right\| .
\end{aligned}
$$

An application of Lemma 4 yields that $b_{n} \rightarrow 0$, as $n \rightarrow+\infty$. Now Lemma 5 implies that $\left\|x_{n+1}-x\right\|+\left\|y_{n+1}-y\right\| \rightarrow 0$, and so $x_{n} \rightarrow x$ and $y_{n} \rightarrow y$ as $n \rightarrow+\infty$. This completes the proof.

Remark 13. Theorems 11 and 12 improve and generalize some known corresponding results.

(i) If $K=H, E=F \equiv I, S(x, y)=s A(y, x)+x-y$, and $T(y, x)=r A(x, y)+x-y$, for all $x, y \in H$, then the problem (11) is equivalent to the problem (13) studied by Petrot [5]. We can get the existence and convergence results of solutions for the problem (13) from Theorems 11 and 12 .

(ii) If $E=F \equiv I, f \equiv 0, S(x, y)=s A(y, x)$, and $T(y, x)=$ $r A(x, y)$, for all $x, y \in H$, then the problem (11) is equivalent to the problem (14) studied by Chang et al. [4]. We can get the existence and convergence results of solutions for the problem (14) from Theorems 11 and 12 .

\section{Conflict of Interests}

The authors declare that there is no conflict of interests regarding the publication of this paper.

\section{Acknowledgments}

This work was supported by the National Natural Science Foundation of China (11301359). This work was also funded by the key program of Xihua University (Grant no. z1312621) and the Project supported by Scientific Research Fund of Sichuan Provincial Education Department (Grant no. 14ZB0130).

\section{References}

[1] C. Lescarret, "Cas d'addition des applications monotones maximales dans un espace de Hilbert," Comptes Rendus de l'Académie des Sciences, vol. 261, pp. 1160-1163, 1965 (French). 
[2] F. E. Browder, "On the unification of the calculus of variations and the theory of monotone nonlinear operators in Banach spaces," Proceedings of the National Academy of Sciences of the United States of America, vol. 56, pp. 419-425, 1966.

[3] I. V. Konnov and E. O. Volotskaya, "Mixed variational inequalities and economic equilibrium problems," Journal of Applied Mathematics, vol. 2, no. 6, pp. 289-314, 2002.

[4] S. S. Chang, H. W. Joseph Lee, and C. K. Chan, "Generalized system for relaxed cocoercive variational inequalities in Hilbert spaces," Applied Mathematics Letters, vol. 20, no. 3, pp. 329-334, 2007.

[5] N. Petrot, "A resolvent operator technique for approximate solving of generalized system mixed variational inequality and fixed point problems," Applied Mathematics Letters, vol. 23, no. 4, pp. 440-445, 2010.

[6] Z. He and F. Gu, "Generalized system for relaxed cocoercive mixed variational inequalities in Hilbert spaces," Applied Mathematics and Computation, vol. 214, no. 1, pp. 26-30, 2009.

[7] H. Nie, Z. Liu, K. H. Kim, and S. M. Kang, "A system of nonlinear variational inequalities involving strongly monotone and pseudocontractive mappings," Advances in Nonlinear Variational Inequalities, vol. 6, no. 2, pp. 91-99, 2003.

[8] R. U. Verma, "Projection methods, algorithms, and a new system of nonlinear variational inequalities," Computers \& Mathematics with Applications, vol. 41, no. 7-8, pp. 1025-1031, 2001.

[9] R. U. Verma, "Generalized system for relaxed cocoercive variational inequalities and projection methods," Journal of Optimization Theory and Applications, vol. 121, no. 1, pp. 203210, 2004.

[10] N. H. Xiu and J. Z. Zhang, "Local convergence analysis of projection-type algorithms: unified approach," Journal of Optimization Theory and Applications, vol. 115, no. 1, pp. 211-230, 2002.

[11] Y. Yao, Y.-C. Liou, and S. M. Kang, "Two-step projection methods for a system of variational inequality problems in Banach spaces," Journal of Global Optimization, vol. 55, no. 4, pp. 801-811, 2013.

[12] Y. J. Cho and X. Qin, "Systems of generalized nonlinear variational inequalities and its projection methods," Nonlinear Analysis: Theory, Methods \& Applications, vol. 69, no. 12, pp. 4443-4451, 2008.

[13] Ya. Alber, "Generalized projection operators in Banach spaces: properties and applications," in Proceedings of the Israel Seminar on Functional Differential Equations, pp. 1-21, Ariel, Israel, 1994.

[14] K.-Q. Wu and N.-J. Huang, "The generalised $f$-projection operator with an application," Bulletin of the Australian Mathematical Society, vol. 73, no. 2, pp. 307-317, 2006.

[15] Y. I. Alber, "Metric and generalized projection operators in Banach spaces: properties and applications," in Theory and Applications of Nonlinear Operators of Accretive and Monotone Type, vol. 178 of Lecture Notes in Pure and Applied Mathematics, pp. 15-50, Dekker, New York, NY, USA, 1996.

[16] J. Fan, X. Liu, and J. Li, "Iterative schemes for approximating solutions of generalized variational inequalities in Banach spaces," Nonlinear Analysis: Theory, Methods \& Applications, vol. 70, no. 11, pp. 3997-4007, 2009.

[17] X. Li, Y.Z. Zou, and N. J. Huang, "On the stability of generalized f-projection operators with an application," Acta Mathematica Sinica, Chinese Series, vol. 53, no. 2, pp. 375-384, 2010.
[18] X. Li, X. S. Li, and N. J. Huang, "A generalized f-projection algorithm for inverse mixed variational inequalities," Optimization Letters, vol. 8, no. 3, pp. 1063-1076, 2014.

[19] L. S. Liu, "Ishikawa and Mann iterative process with errors for nonlinear strongly accretive mappings in Banach spaces," Journal of Mathematical Analysis and Applications, vol. 194, no. 1, pp. 114-125, 1995.

[20] H.-Y. Lan, J. H. Kim, and Y. J. Cho, "On a new system of nonlinear $A$-monotone multivalued variational inclusions," Journal of Mathematical Analysis and Applications, vol. 327, no. 1, pp. 481-493, 2007.

[21] R. U. Verma, "General convergence analysis for two-step projection methods and applications to variational problems," Applied Mathematics Letters, vol. 18, no. 11, pp. 1286-1292, 2005.

[22] S. B. Nadler Jr., "Multi-valued contraction mappings," Pacific Journal of Mathematics, vol. 30, pp. 475-488, 1969. 


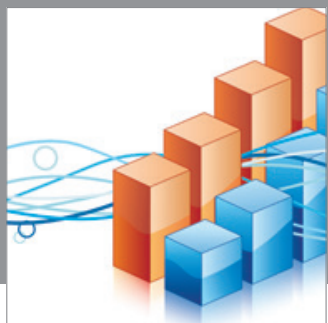

Advances in

Operations Research

mansans

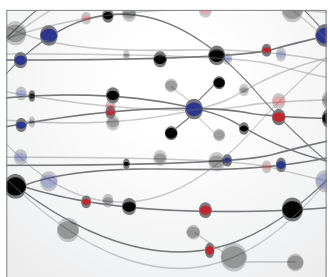

The Scientific World Journal
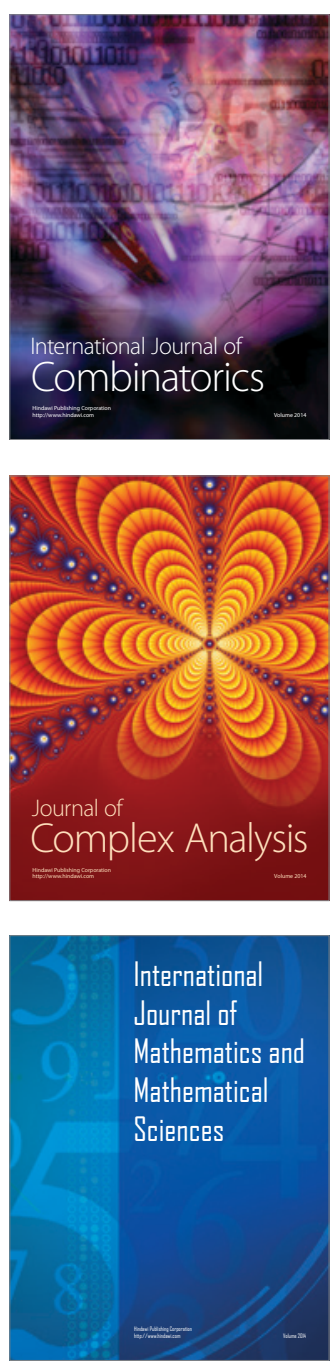
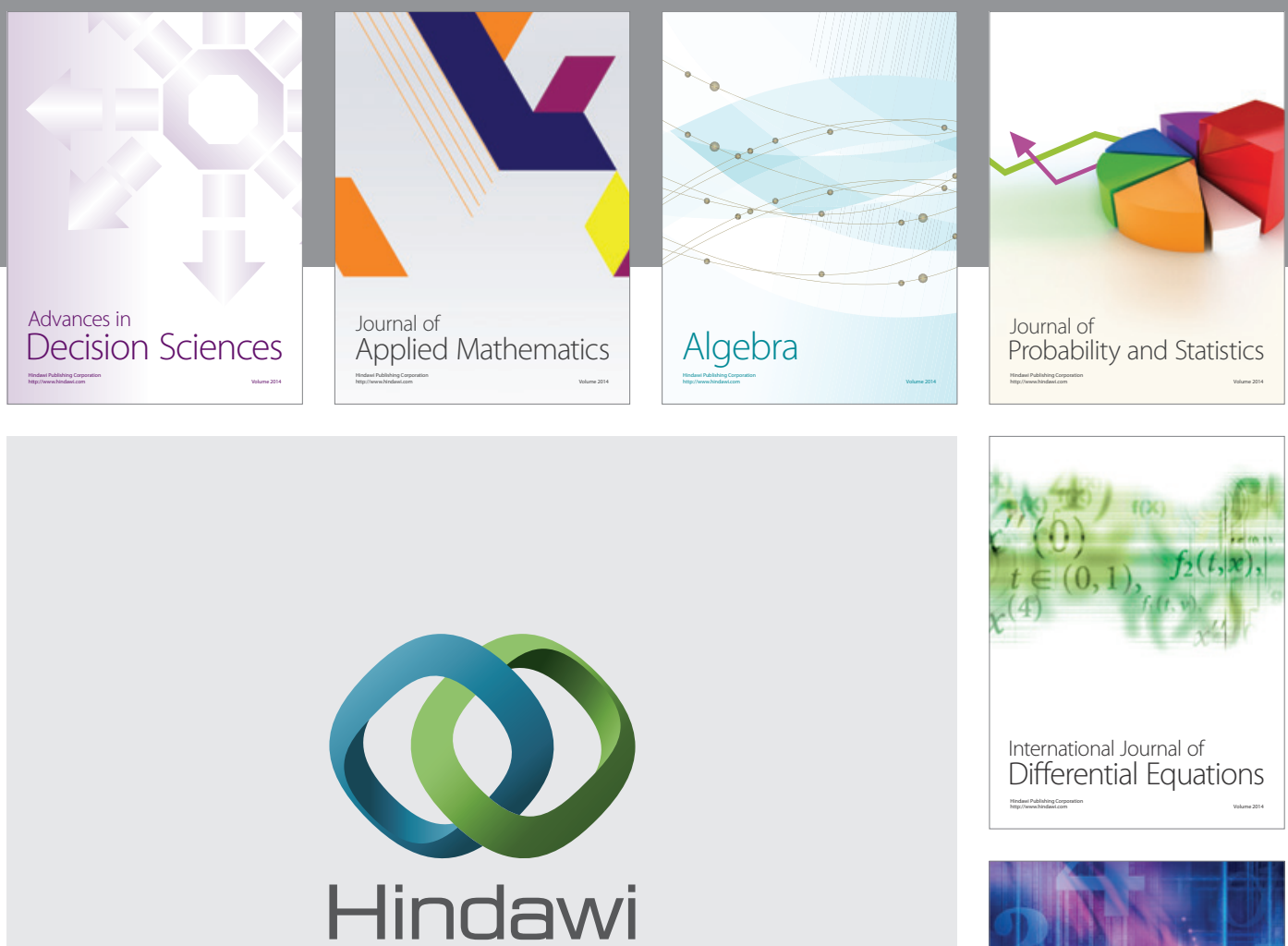

Submit your manuscripts at http://www.hindawi.com
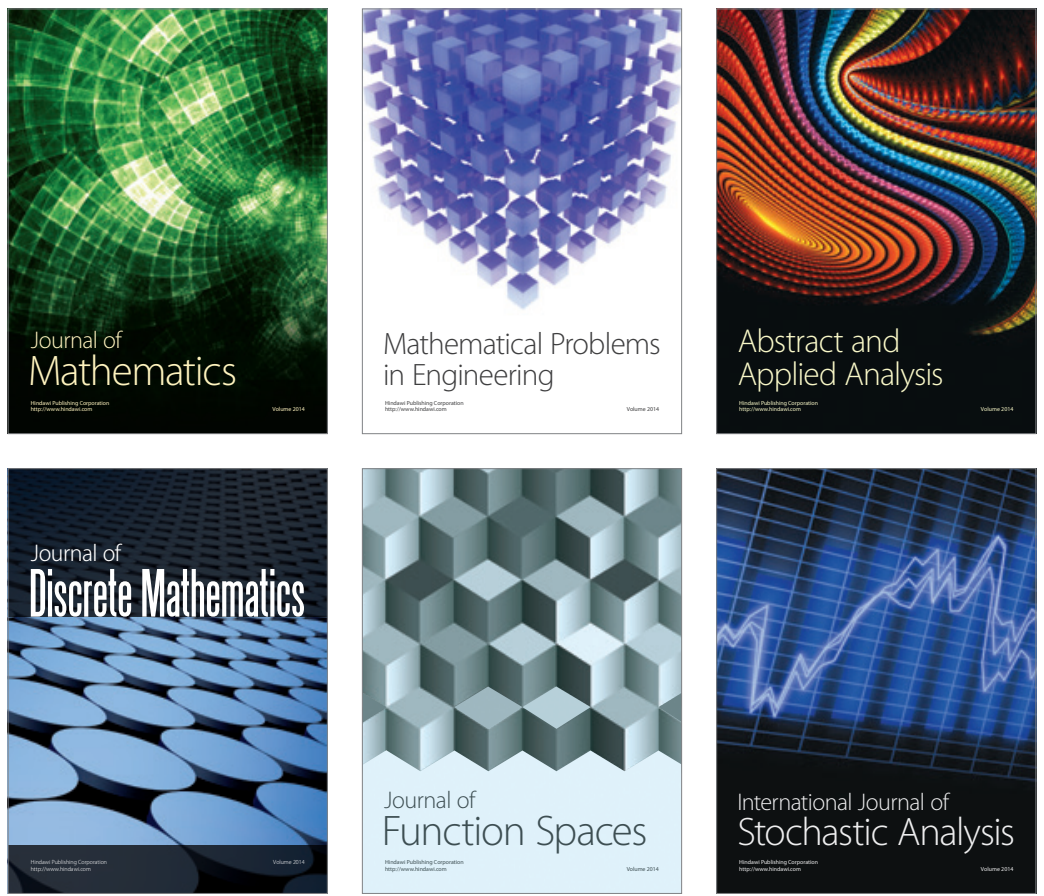

Journal of

Function Spaces

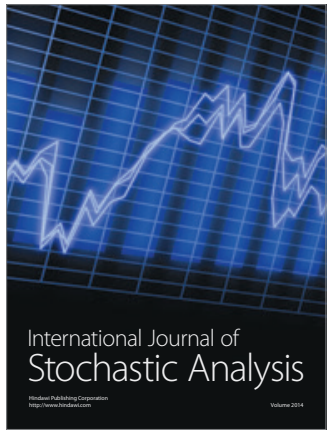

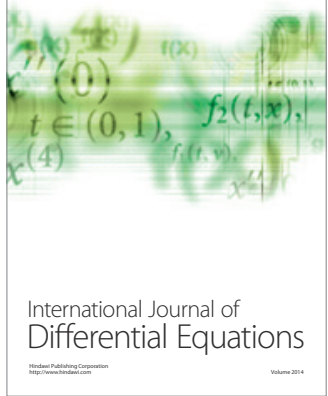
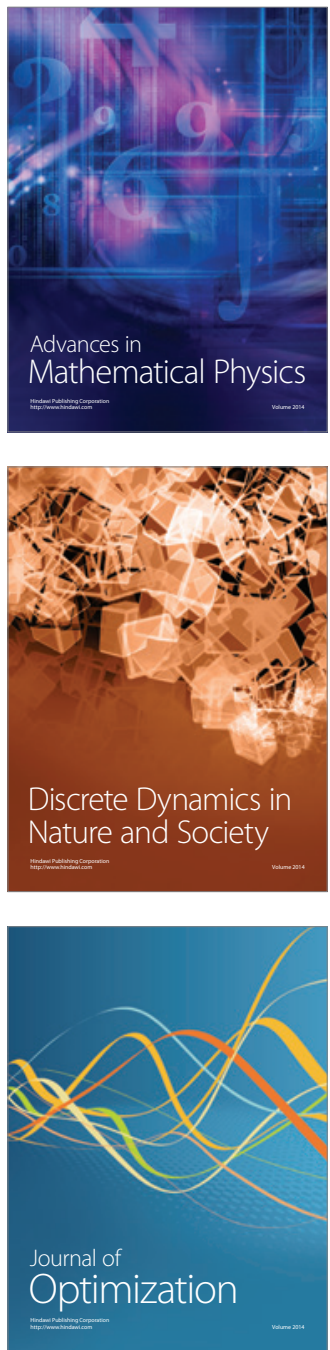